\title{
Determination of the minimally important difference (MID) in multi-biomarker disease activity (MBDA) test scores: impact of diurnal and daily biomarker variation patterns on MBDA scores
}

\author{
David Chernoff ${ }^{1}$ - P. Scott Eastman ${ }^{1} \cdot$ Ching Chang Hwang ${ }^{1} \cdot$ Darl D. Flake $I^{2} \cdot$ Xingbin Wang $^{1} \cdot$ Alan Kivitz $^{3}$. \\ Jeffrey R. Curtis ${ }^{4}$
}

Received: 10 January 2018 / Revised: 16 August 2018 / Accepted: 20 August 2018 / Published online: 29 August 2018

(C) The Author(s) 2018

\begin{abstract}
The Multi-Biomarker Disease Activity (MBDA) score is a validated rheumatoid arthritis (RA) disease activity measure based on 12 serum biomarkers. Here, we evaluate short-term biological variability of MBDA scores to determine the magnitude of change that might be considered clinically meaningful. Twenty-eight adult seropositive RA patients with clinically stable disease and no changes in RA medications for 4 weeks prior to study were enrolled. Nine serum samples were obtained over four consecutive days (non-fasting). MBDA score variation was assessed day-to-day (daily) and within $24 \mathrm{~h}$ (diurnal). The standard deviation (SD) of MBDA scores was calculated by a linear mixed model including random effects for patient, day, and time of day. The

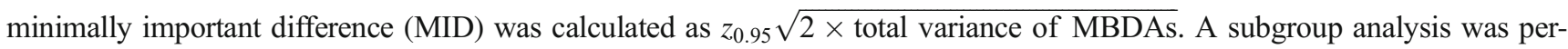
formed for patients with active RA (moderate or high MBDA score). The SD of MBDA score change in the full cohort was 4.7 in a combined daily-diurnal variation analysis, which corresponds with an MID of 11. The SD of MBDA score change in the subset of patients with active RA (moderate/high MBDA scores) was 3.6. This corresponds with an MID of 8 units in patients with active RA for whom clinicians are most likely to need guidance with respect to therapeutic decisions. Changes in MBDA score $\geq$ 8 represent a change in RA disease activity that clinicians can use as a benchmark for therapeutic drug efficacy and can be incorporated into a treat-to-target strategy.
\end{abstract}

Keywords Minimally important difference (MID) · Multi-biomarker disease activity (MBDA) $\cdot$ Rheumatoid arthritis $\cdot$ Vectra DA

\section{Introduction}

Rheumatoid arthritis (RA) is a chronic inflammatory disease of unknown etiology in which joint damage and physical disability are major adverse outcomes resulting in poor quality of life and premature mortality. Successful treatment of RA attains a state of low disease activity and/or clinical remission to

David Chernoff

dchernoff@crescendobio.com

1 Crescendo Bioscience Inc., 341 Oyster Point Blvd, South San Francisco, CA, USA

2 Myriad Genetics, Inc., 320 Wakara Way, Salt Lake City, UT, USA

3 Altoona Center for Clinical Research, 1125 Old Rte 220 N, Duncansville, PA, USA

4 The University of Alabama at Birmingham, 510 20th Street S, Birmingham, AL, USA prevent progressive joint destruction and maintaining functional capabilities. Three major classes of disease-modifying anti-rheumatic drugs (DMARDs) have demonstrated efficacy in RA: conventional synthetic DMARDs (csDMARDs, e.g., methotrexate, leflunomide), targeted synthetic DMARDs (tsDMARDs, e.g., tofacitinib), and biological agents (bDMARDS, e.g., etanercept, adalimumab, infliximab) [1]. An international rheumatology task force has recommended a Treat-to-Target approach to achieve optimal therapeutic outcomes by using defined measures of clinical disease activity to guide treatment in order to achieve remission or a low disease activity state [2]. Both the American College of Rheumatology (ACR) and the European Union League Against Rheumatism (EULAR) recommendations that the management of RA include systematic longitudinal and quantitative RA disease activity assessment $[3,4]$.

There are many measures available to assess RA disease activity. These include several "composite" disease activity 
indices primarily composed of "clinical phenotypes," tender and swollen joint counts (TJC and SJC, respectively), physician global assessment, and patient global assessment. Commonly used composite measures include the clinical disease activity index (CDAI) [5], the disease activity score (DAS) with acute phase reactants included (DAS28-ESR, DAS28-CRP) [6], a simplified disease activity index (SDAI) [7], and the Routine Assessment of Patient Index Data 3 (RAPID3) [8]. Another validated measure of RA disease activity is the multi-biomarker disease activity (MBDA) score, which is an objective molecular measure based on an algorithmic assessment of 12 serum biomarkers [9, 10]. Most RA disease activity measures categorize the amount of disease activity and can be used to inform treatment decisions at a single point in time. For example, the MBDA score is reported on a scale of 1-100 with categories for high (> $44)$, moderate $(30-44)$, and low $(<30)$ disease activity. Patients in the high or moderate categories are considered to have active disease and may be appropriate for treatment intensification. For use in a Treat-to-Target approach, clinicians must be able to determine whether changes in a score over time are representative of a true change in disease activity. This requires that the short-term variability of disease activity measures be well characterized.

Short-term variability is indicative of inter-observer precision for composite disease activity indices or laboratory and/ or biologic variability of biomarkers for molecular measures of disease activity. Only changes in scores that are greater than the short-term variability of a disease activity measure can be reliably correlated with true clinical changes in order to inform treatment decisions. Previous studies have evaluated the shortterm variation in many composite clinical measures of disease activity in patients with clinically stable disease [11-13]. In addition, the laboratory variability of the MBDA score has been well characterized, including pre-analytical effects of blood sampling/handling, precision of the biomarker assay, and precision of the MBDA score $[14,15]$. Although these previous studies have shown that this laboratory variation is minimal, the biologic variability of the individual biomarkers included in the MBDA score has not been assessed. For patients with RA, previous reports have shown short-term biological variations in individual biomarkers that appear to have exaggerated circadian rhythms or diurnal variation such as IL$6[16,17]$. Determining the short-term biologic variability is therefore critical to characterizing the minimally important difference (MID) of the MBDA score in order to determine whether changes in score represent meaningful differences in a patient's disease activity.

In this study, we determined the MID for the MBDA score. This was done by evaluating short-term biological variation of the MBDA score over a 24-h period (diurnal) and from day to day (daily) in RA patients on stable DMARD therapy. In order to assess the MID in patients with clinically active disease for whom clinicians are most likely to need guidance with respect to therapeutic decisions, a subgroup analysis was performed in patients with moderate to high disease activity at baseline.

\section{Materials and methods}

\section{Study design and enrollment criteria}

This prospective observational study was conducted at a single rheumatology clinical research center-the Altoona Center for Clinical Research (Duncansville, PA, USA). Serum samples were collected at multiple time points over 4 days. This time period allowed for diurnal variations in MBDA score to be evaluated over the first $24 \mathrm{~h}$ and daily variations to be evaluated over a period of multiple successive days.

Inclusion criteria were as follows: RA diagnosed using the ACR 2010 Revised Classification Criteria [18], age 21 to 80 years, positive blood tests(s) for rheumatoid factor (RF) and/or anti-cyclic, citrullinated peptide antibodies (CCP or ACPA), and clinically stable RA, defined as having received csDMARD, tsDMARD, and/or bDMARD treatment for greater than 8 weeks with no medication change in the 4 weeks prior to enrollment. NonDMARD medication use (e.g., glucocorticoids, NSAIDs) was also obtained at screening. There was no restriction on NSAID use, and corticosteroid use was allowed where the daily dose did not exceed $10 \mathrm{mg}$ /day with no change in the 4 weeks prior to enrollment. Patients were screened up to 15 days in advance of enrollment, and screening results were provided to the investigator 10 days prior to enrollment. Patients with low, moderate, or high clinical disease activity as defined by CDAI, SDAI, or DAS28ESR were included in the study $[5,7,19]$. Subject participation was timed so that subjects receiving parenteral biologic therapy would not have a scheduled dose during the 4-day study period.

Patients were excluded if they had a history of/or current inflammatory joint disease other than RA or other systemic autoimmune disorders, known active or chronic infection of any kind or concomitant malignancies or previous malignancies in the last 4 years. Patients receiving tocilizumab or those taking opioids 7 days prior to and during study visit (with the exception of subjects with high disease activity) were also excluded. Patients taking opioids were excluded in order to avoid confounding of clinical and patientreported outcomes regarding pain, which might be masked by patients taking opiates at the time of the clinical assessment of disease activity. Eligible subjects were enrolled by the rheumatologist study investigator. The protocol received Investigational Review Board Approval, and all patients provided informed consent. 


\section{Serum collection and biomarker measurement}

Patients were admitted to the research center for an overnight stay on day 1 , and serum samples were collected at 8 a.m., 12 p.m. (noon), 4 p.m., 8 p.m., and 12 a.m. (midnight) during day 1 , at 8 a.m. and 12 p.m. (noon) on day 2. Patients were released after the noon serum sample was collected on day 2 and returned to the site for serum sample collection at a single time point ( 8 a.m.) on day 3 and day 4 . All 8 a.m. samples were non-fasting. A schedule of sample collection is presented in Fig. 1.

Clinical data collected included patient global assessment of pain activity, patient global assessment of disease activity, physician global assessment, swollen/tender joint examination, CDAI, RAPID3, and Health Assessment Questionnaire (HAQ). Clinical disease activity measurements were collected daily at each 8 a.m. sample collection.

Samples were collected as previously described for the MBDA test (Crescendo Bioscience, South San Francisco, CA, USA) [14]. Briefly, serum was collected in $4 \mathrm{~mL}$ BD $\mathrm{SST}^{\mathrm{TM}}$ transport tubes, mixed by gently inverting $4-5$ times, allowed to clot upright at room temperature for 30-45 min, and then centrifuged for $15 \mathrm{~min}$ at 1000 to $1300 \mathrm{RCF}$ in a swing bucket centrifuge at room temperature. Centrifuged BD SST Transport tubes were refrigerated $\left(2-8^{\circ} \mathrm{C}\right)$ for up to $78 \mathrm{~h}$ following collection, then shipped in a designated temperature-controlled container to the testing laboratory and frozen at $-80^{\circ} \mathrm{C}$ until analysis.

Single archived de-identified frozen serum samples were tested in a random manner at the testing laboratory (Crescendo Bioscience, South San Francisco, CA). The laboratory is certified under the CMS Clinical Laboratory Improvement Amendments and accredited by the College of American Pathologists. Biomarkers were measured by electroluminescence-based multiplex immunoassays on the Meso Scale Discovery Multi-Array platform (Meso Scale Discovery, Bethesda, MD, USA) [15].

Twelve biomarkers were measured in serum samples as previously described [15] and included C-reactive

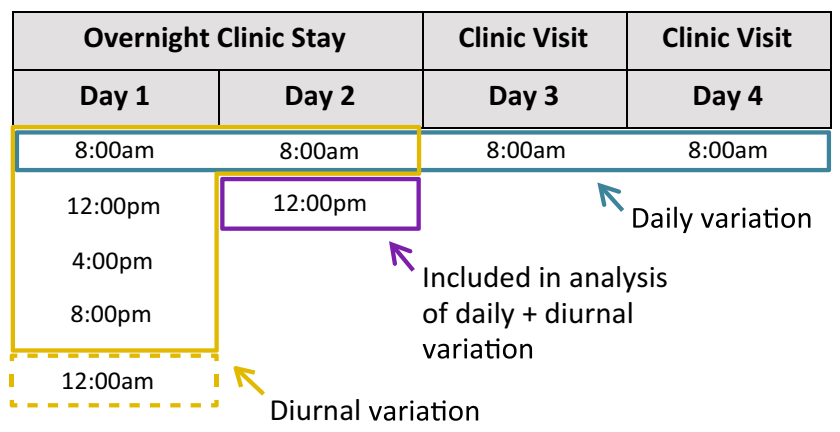

Fig. 1 Study design. Blood sample collection schedule indicating time points for diurnal variation analysis and daily variation analysis for all patients. For the subgroup analysis of moderate to high MBDA score patients, the 12 a.m. midnight time point was excluded protein (CRP), epidermal growth factor (EGF), leptin, interleukin 6 (IL-6), matrix metalloproteinase-1 (MMP1), matrix metalloproteinase-3 (MMP-3), resistin, serum amyloid A (SAA), tumor necrosis factor receptor-1 (TNFR-I, TNFRSF1A), vascular cell adhesion molecule1 (VCAM-1), vascular endothelial growth factor A (VEGF-A), and human cartilage glycoprotein-39 (YKL40). The MBDA score was calculated for each sample using a validated algorithm $[9,10,20]$. MBDA scores were categorized as low $(<30)$, moderate $(30-44)$, or high (>44) [10].

\section{Statistical analysis}

Twenty-eight patients were enrolled in the study to investigate diurnal and daily variation. The mean and standard deviation (SD) of the MBDA score was calculated for the patients in each MBDA category based on their scores at the baseline (day 1, 8 a.m.) and over the next three consecutive days. Daily and diurnal variation was calculated using a linear random-effects model. The patient, day, and time of day were included as predictors of MBDA score. The daily, diurnal, and unexplained variability were summed to calculate the total variability of the MBDA score. In the sub-group analyses for the patients with a moderate to high MBDA score at baseline, the 12 a.m. midnight time point was excluded as serum sampling in routine clinical practice would not be expected to occur in the middle of the night. MID was calculated as

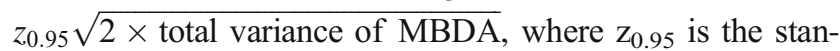
dard normal deviate corresponding to the 95 th percentile (90\% confidence interval) [21]. The MID is the 90\% upper confidence limit for the standard deviation of MBDA scores that are different due to chance (short-term variability, including laboratory variability), such that $90 \%$ of patients whose MBDA score changes by less than the MID have no change in disease activity. This threshold was selected in order to minimize the proportion of patients with a true change in RA disease activity whose change in MBDA score is interpreted as short-term variability (i.e., false negatives). The MID represents the largest expected change in MBDA score due to chance. All analyses were performed in R (version 3.2.4).

\section{Results \\ Patient characteristics}

Baseline demographic data for the 28 eligible, enrolled patients are presented in Table 1. All categories of MBDA disease activity scores were represented at baseline with 6 patients $(21.4 \%)$ having a low MBDA score, 13 patients $(46.4 \%)$ having a moderate MBDA score, and 
Table 1 Baseline clinical characteristics

\begin{tabular}{|c|c|}
\hline Baseline characteristic & Median $(\mathrm{Q} 1, \mathrm{Q} 3)$ or $n(\%)$ \\
\hline Age (years) & $66(57,70)$ \\
\hline Sex (female) & $18(64.3 \%)$ \\
\hline Body mass index $\left(\mathrm{kg} / \mathrm{m}^{2}\right)$ & $31.0(25.1,35.2)$ \\
\hline Race (Caucasian) & $28(100 \%)$ \\
\hline Medication $^{\mathrm{a}}$ & $n(\%)$ \\
\hline Non-biologic DMARD & $27(96.4 \%)$ \\
\hline MTX & $26(92.9 \%)$ \\
\hline Biologic & $8(28.6 \%)$ \\
\hline TNFi (etanercept, certolizumab, infliximab, adalimumab) & $7(25 \%)$ \\
\hline $\mathrm{TNFi}+\mathrm{MTX}$ & $6(21.4 \%)$ \\
\hline Non-TNFi (rituximab) & $1(4 \%)$ \\
\hline Clinical assessment & Median (Q1, Q3) \\
\hline Swollen Joint Count (SJC28) & $4.5(0,10)$ \\
\hline Tender Joint Count (TJC28) & $4.5(0,15.5)$ \\
\hline \multicolumn{2}{|c|}{ Global Assessment Disease Activity $(0=$ patient doing very well; $10=$ patient doing very poorly) } \\
\hline Physician & $2.0(0.8,3.5)$ \\
\hline Patient & $2.5(1.0,5.0)$ \\
\hline Patient Assessment of Pain ( $0=$ no pain; $100=$ most severe pain $)$ & $26.0(9.5,55.0)$ \\
\hline Routine Assessment of Patient Index Data 3 (RAPID3) & $6.5(3.7,14.2)$ \\
\hline Multi-Biomarker Disease Activity (MBDA) & $39.5(33.0,48.0)$ \\
\hline Clinical Disease Activity Index (CDAI) & $15.8(4.5,35.8)$ \\
\hline
\end{tabular}

${ }^{a}$ Patients who took multiple medications could be counted more than once for $\%$ calculations

9 patients $(32.1 \%)$ having a high MBDA score. The median age of patients was 66 years, $64 \%$ of patients were female, and $100 \%$ of patients were Caucasian (Table 1).

All patients maintained stable DMARD therapy throughout the study. Ninety-three percent of patients were receiving methotrexate (MTX) therapy while only $29 \%$ of patients were receiving biologic therapy and none were taking prednisone or other glucocorticoids. The median number of joint counts was 4.5 for both swollen and tender joint counts. Median CDAI (15.8), RAPID3 (6.5), and MBDA (39.5) scores correspond with moderate disease activity; however, all three levels of disease activity were represented as measured by baseline MBDA scores. No patients received infusions or injections of their biologic agent during the conduct of the study. The mean and SD of the clinical measures over time are presented in Fig. 2.

\section{Daily effects on MBDA score}

The mean and SD of the MBDA score for the patients in each MBDA category at each time point are presented in Fig. 3. The means of the MBDA score were stable within the moderate and high categories, but showed more fluctuation within the low MBDA category over time.

\section{Diurnal effects on MBDA score}

Diurnal variation of the MBDA score was evaluated by obtaining serum samples at seven time points in a $24-\mathrm{h}$ period. The mean MBDA score and SD were calculated for the

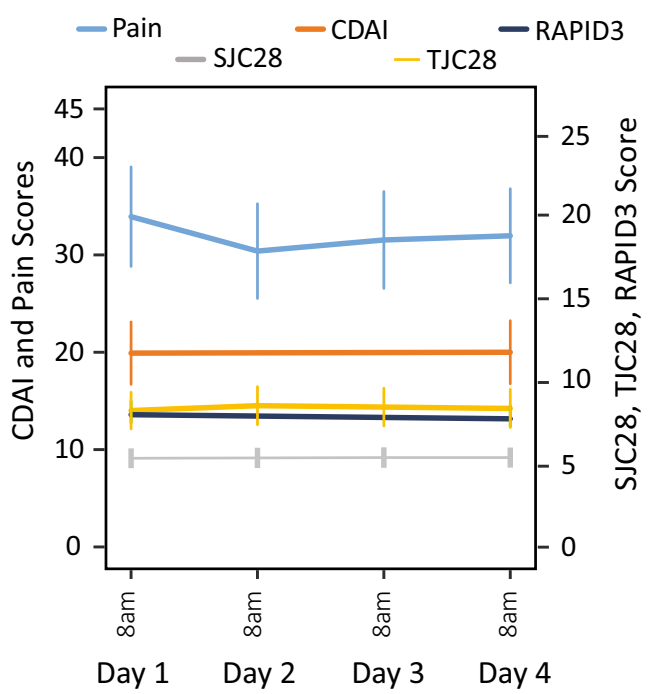

Fig. 2 Daily variation of clinical measures (mean, standard error) 


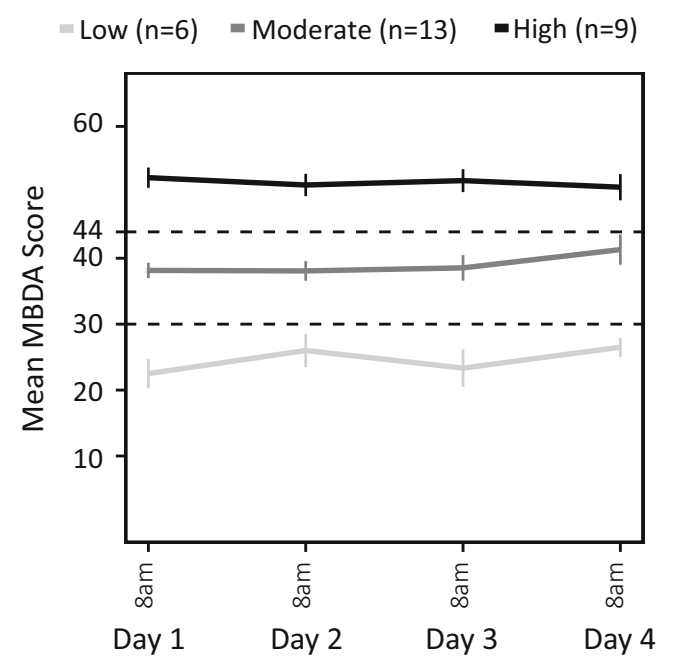

Fig. 3 Daily variation by baseline MBDA disease activity category

patients in each MBDA category based on their scores at the different time points. For graphical purposes, both the diurnal (within a day) and the daily ( 8 a.m. for each of 4 days) observations for the MBDA score for all three disease activity categories are presented in Fig. 4.

MBDA scores throughout the day remained constant; however, scores began to increase in the evening, particularly at 12 a.m. and then returned to their daily average. This was readily apparent in all three disease activity categories, but was most pronounced in the low disease activity group (Fig. 4). Inspection of the concentrations of the individual biomarkers indicates the sources of the diurnal variance observed in the MBDA score (Fig. 5). Both IL-6 and leptin concentrations increased while EGF concentrations diminished. As EGF is inversely correlated with disease activity while IL-6

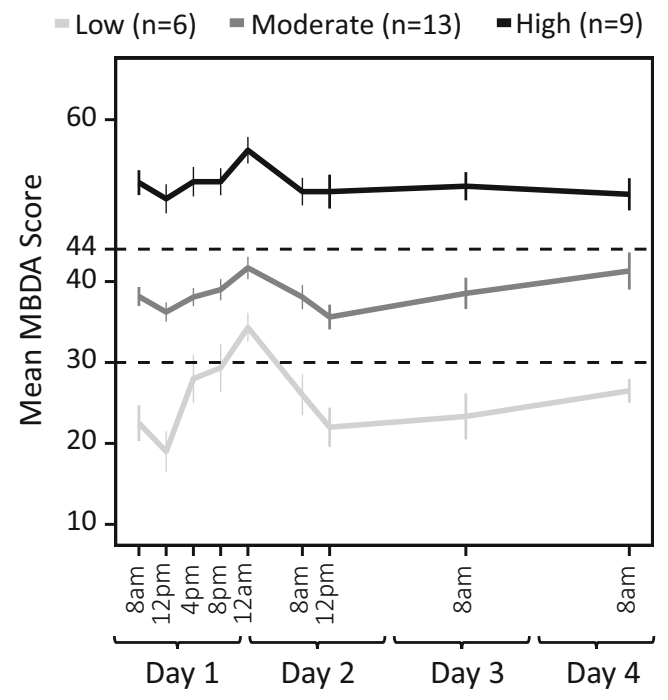

Fig. 4 Daily and diurnal variation by baseline MBDA category and leptin are positively correlated, an increase in the MBDA score would be expected.

\section{Determination of MID}

A linear model of MBDA including random effects for patient, day, and time of day for all time points reflects the changes of the MBDA score over time. In a combined dailydiurnal variation analysis including all patients the SD of MBDA score change was 4.7 and the MID was 11 . In the subset analysis of patients with active RA (moderate/high disease activity categories, $n=22$ ), the total SD of MBDA scores was 3.6, and the MID was therefore $8 \mathrm{MBDA}$ units. When the MBDA scores were adjusted for age, sex, and adiposity [22], the MID remained unchanged at 8 units.

\section{Discussion}

Treat-to-Target approaches have demonstrated efficacy in RA treatment [2]; however, this approach requires RA disease activity to be accurately quantified in order to differentiate significant response to therapy from loss of therapeutic effect. Early in the understanding of RA pathogenesis, only clinical phenotypes were available for discerning the extent of disease activity including estimation of the number of swollen joints or tender joints and the physician's overall global assessment. However, formal joint counts are often not performed in routine clinical practice settings [23-25] and have been shown to be poorly reproducible $[26,27]$. Although some composite indices incorporate clinical and biomarker estimates of disease activity, these measures (CRP, erythrocyte sedimentation rates) are normal in up to $70 \%$ of patients with active disease [28] and lack the sensitivity to accurately detect and quantitate disease activity. Ultimately, none of these composite clinical measures have been proven to predict further joint damage for individual patients and continued structural damage has been observed in patients in clinical remission as determined by these indices [29]. In contrast, the MBDA test was developed to quantitate molecular disease activity in order to account for the heterogeneous biologic phenotypes that drive RA disease activity [15]. This objective measure of disease activity has been shown to predict permanent joint damage based on radiographic progression [30-33].

Determining underlying short-term biologic variability of the MBDA score is critical for appropriate use of the test in clinical practice. This allows clinicians to distinguish true changes in RA disease activity from variability within the measure. Here, we report on the MID of the MBDA score, which by design incorporates both sample 

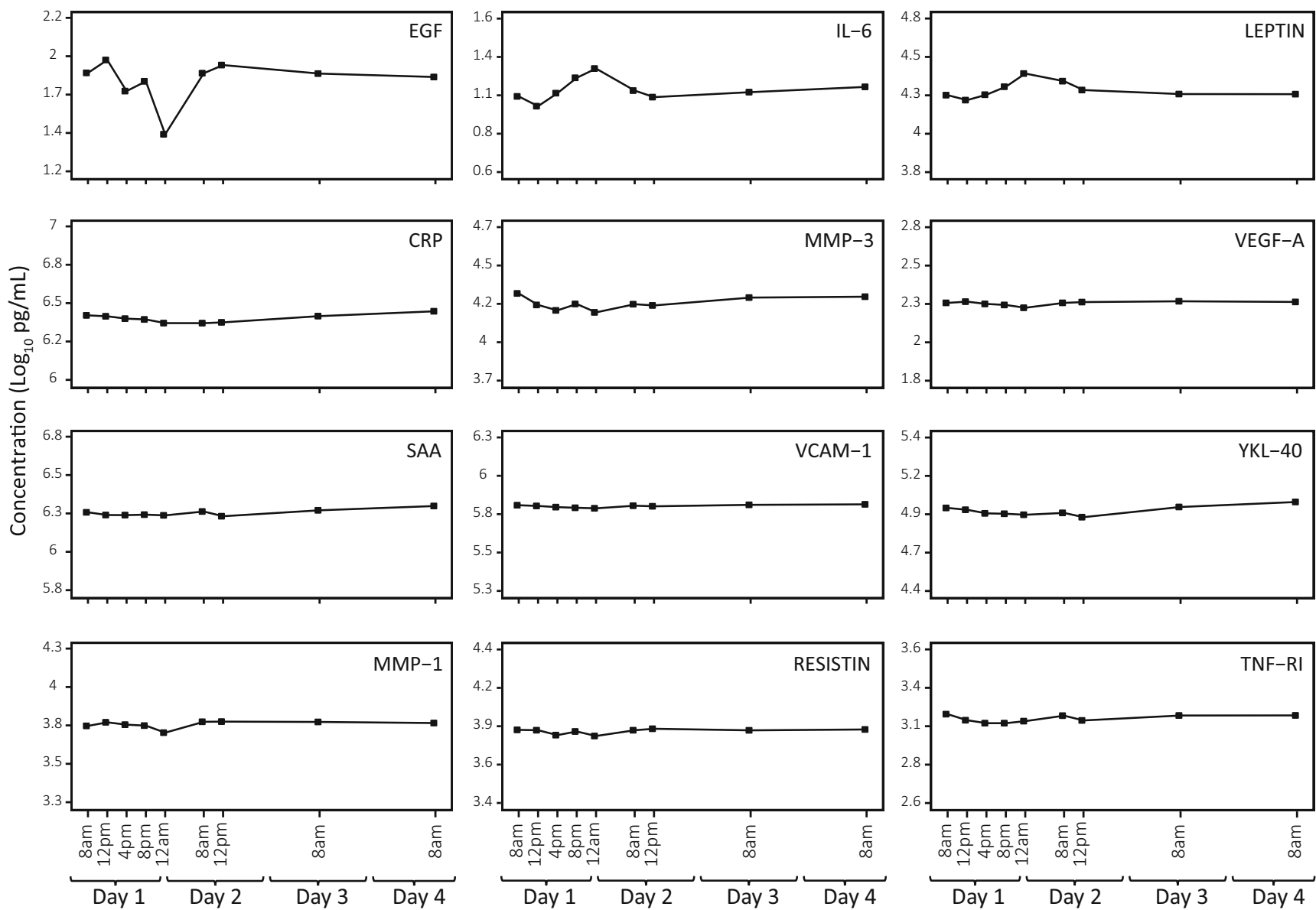

Fig. 5 Individual biomarkers in clinically stable patients diurnal and daily variation

handling and assay variability as well as daily and diurnal biologic variability of the biomarkers.

The amount of daily and diurnal variability for the MBDA score in all patients was very low, at only 4.7 score units, and the corresponding MID was 11 units. This indicates that for patients in any MBDA disease activity category (low, moderate, high), a MBDA score change of at least 11 is necessary to be considered clinically significant. However, RA is likely well controlled in patients with low disease activity and relative changes in MBDA score are unlikely to prompt a change in treatment regimen. In contrast, patients with moderate to high MBDA scores are often either initiating or not responding to DMARD therapy. As such, this subset of patients represents those with clinically active disease for whom clinicians are most likely to need guidance in therapeutic decision making. Among patients with moderate or high disease activity, the daily-diurnal variability was 3.6 units and the MID was 8 . In this patient population, a change in MBDA score greater than or equal to 8 corresponds with true changes in disease activity.

The difference between the MID for all patients (low, moderate, or high disease activity) compared to those with active RA (moderate or high disease activity) can be explained by inspection of the diurnal performance of the individual biomarkers. Three biomarkers, IL-6, leptin, and EGF, vary in their concentrations throughout the day (Fig. 5) but primarily peak at night (IL-6 and leptin) or hit their nadir as in the case of EGF. While IL-6 and leptin concentrations increase resulting in a temporary increase in the MBDA score, EGF, however, is inversely correlated with RA disease activity and would also be expected to increase the MBDA score. Similar diurnal variations for these biomarkers have been previously described [16, 17, 34-36]. At higher disease activity levels, the additional nine biomarkers contribute to the high score lessening the total impact of the diurnal variation of IL-6, EGF, and leptin, whereas at low disease activity levels, all of the diurnal increase in the MBDA is resultant from three biomarkers who are known to have a diurnal pattern.

The MID of 8 enables clinicians to identify which patients with active RA (moderate or high disease activity) demonstrate a meaningful decrease in disease activity (MBDA score decrease $\geq 8$ ), no change in disease activity (MBDA score change of $<8$ ), or an increase in disease activity (MID score increase $\geq 8$ ). This is clinically relevant, as MBDA is a continuous variable in which significant changes in disease activity can be observed without any corresponding change in the 
disease category. For example, an MBDA score decrease from 65 to 50 does not result in a change in disease activity category; however, this change is greater than the MID and would represent a true decrease in disease activity. Conversely, an MBDA score change from 46 to 43 represents a change in category (high to moderate) but does not represent a true decrease in disease activity (change $<8$ ). In the Treat-to-Target approach, the ability to distinguish true longitudinal changes in disease activity that exceed measurement error and short term biologic variability is critical for clinicians to make appropriate treatment decisions and improve therapeutic response.

While the short-term variability for laboratory-based measures is the MID, variability of composite clinical measures has been evaluated using several metrics. These measures of variability have been well established in the literature and are used to identify true changes in disease activity. It is noteworthy that the MID for the MBDA score reported here correlates well with the short-term variability reported for other measures of RA disease activity. van Gestel et al. estimated a measurement error of 0.6 DAS units using inter-period correlation matrix analysis and discriminant validity [13] resulting in a change from baseline of 1.2 units required to exceed assay noise (DAS range, 1-10 units). Heegard et al. [11] determined a least significant difference $(\mathrm{LSD}=1.96 \times \mathrm{SD})$ from 30 patients with clinic visits 1 week apart for DAS28-CRP as 0.8 DAS units (DAS28-CRP range, 1-9.6 units). Because the MBDA score was initially developed by correlation with the DAS, it can therefore be converted to the same scale [15]. The MID of 8 for patients with high or moderate disease activity correspond to 0.66 DAS28-CRP, representing a comparable or improved short-term variability relative to DAS.

Although the MBDA score cannot be directly converted to other composite measures of disease activity, previous studies have demonstrated very similar measurement errors for DAS, CDAI, and SDAI. The LSD was determined to be 8.4 units for CDAI and 8.3 units for SDAI (CDAI and SDAI ranges, 0-76). In a test-retest study by Uhlig et al. where patients were evaluated at baseline and 5-7 days later, the smallest detectable difference $(\mathrm{SDD}=1.96 \times \mathrm{SD})$ was $1.32 \mathrm{DAS}$ units for DAS28-ESR, 8.26 units for SDAI, 8.05 units for CDAI, and 1.48 for RAPID3 (RAPID3 range, 0-30) [12]. For patient reported outcomes, the minimal clinically important difference (MCID) or minimal clinically important improvement (MCII) can also be evaluated. Ward et al. report an MCID of 1.0 for DAS28-CRP, 1.2 for DAS28-ESR, 13 for SDAI, and 13 for CDAI [37]. Interestingly, Curtis et al. report MCID cut points for CDAI improvement as 12 units for high disease activity, 6 for moderate disease activity, and 1 for low disease activity (CDAI < 10) [38]; the MCID for worsening among people doing well in low disease activity was $>2$ units. To this point, the MID of the MBDA for disease worsening (among people starting in low disease activity or remission) may be different than the MID of 8; however, determining a MID for worsening of the MBDA could not be assessed in the current study given the small sample size ( $n=6$ in low disease activity at baseline). Moreover, we would differentiate an MID, which is typically based on mathematical estimates of variability measured in stable patients over short periods of time, from an MCID, which is clinically anchored. Estimating the MID of the MBDA, as well as its MCID, for RA patients in low disease activity remains a topic for future investigation.

In summary, Treat-to-Target recommendations improve the management of RA in clinical practice and achieve optimal therapeutic outcomes by closely monitoring a patient's disease activity [2]. The MBDA score provides an objective, molecular measure of disease activity; however, clinicians must be able to differentiate true changes in disease activity from short-term biologic variability in order to utilize MBDA in a Treat-to-Target approach. Here, we determined that changes in MBDA score greater than or equal to 8 represent true changes in disease activity for patients with active RA, while changes less than 8 represent biologic variability of the biomarkers. To this end, the knowledge of diurnal and daily variation of the MBDA score and defined MID should be helpful to the practicing rheumatologist in their decision-making and patient care when using MBDA test.

Funding This study was financially supported by Crescendo Bioscience, Inc.

\section{Compliance with ethical standards}

Conflict of interest D. Chernoff Shareholder of: Myriad Genetics, Inc., Employee of: Crescendo Bioscience Inc., P. S. Eastman Shareholder of: Myriad Genetics, Inc., Employee of: Crescendo Bioscience Inc., C. C. Hwang Shareholder of: Myriad Genetics, Inc., Employee of: Crescendo Bioscience Inc., D. Flake Shareholder of: Myriad Genetics, Inc., Employee of: Myriad Genetics, Inc., X. Wang Shareholder of: Myriad Genetics, Inc., Employee of: Crescendo Bioscience Inc., A. Kivitz Consultant for: Genentech, Pfizer, UCB, Janssen, Novartis Abvie, J. Curtis Grant/research support from: Crescendo Bioscience Inc., Consultant for: Crescendo Bioscience Inc.

Open Access This article is distributed under the terms of the Creative Commons Attribution 4.0 International License (http:// creativecommons.org/licenses/by/4.0/), which permits unrestricted use, distribution, and reproduction in any medium, provided you give appropriate credit to the original author(s) and the source, provide a link to the Creative Commons license, and indicate if changes were made.

\section{References}

1. Smolen JS, Landewé R, Bijlsma J, Burmester G, Chatzidionysiou K, Dougados M, Nam J, Ramiro S, Voshaar M, van Vollenhoven R, Aletaha D, Aringer M, Boers M, Buckley CD, Buttgereit F, Bykerk V, Cardiel M, Combe B, Cutolo M, van Eijk-Hustings Y, Emery P, Finckh A, Gabay C, Gomez-Reino J, Gossec L, Gottenberg JE, Hazes JMW, Huizinga T, Jani M, Karateev D, Kouloumas M, Kvien T, Li Z, Mariette X, McInnes I, Mysler E, Nash P, Pavelka 
K, Poór G, Richez C, van Riel P, Rubbert-Roth A, Saag K, da Silva J, Stamm T, Takeuchi T, Westhovens R, de Wit M, van der Heijde D (2017) EULAR recommendations for the management of rheumatoid arthritis with synthetic and biological disease-modifying antirheumatic drugs: 2016 update. Ann Rheum Dis 76(6):960-977

2. Smolen JS, Aletaha D, Bijlsma JW, Breedveld FC, Boumpas D, Burmester $\mathrm{G}$ et al (2010) Treating rheumatoid arthritis to target: recommendations of an international task force. Ann Rheum Dis 69(4):631-637

3. Singh JA, Saag KG, Bridges SL Jr, Akl EA, Bannuru RR, Sullivan MC et al (2015) American College of Rheumatology Guideline for the treatment of rheumatoid arthritis. Arthritis \& rheumatology (Hoboken, NJ). 2016 68(1):1-26

4. Smolen JS, Landewe R, Breedveld FC, Buch M, Burmester G, Dougados $\mathrm{M}$ et al (2014) EULAR recommendations for the management of rheumatoid arthritis with synthetic and biological disease-modifying antirheumatic drugs: 2013 update. Ann Rheum Dis 73(3):492-509

5. Aletaha D, Nell VP, Stamm T, Uffmann M, Pflugbeil S, Machold K et al (2005) Acute phase reactants add little to composite disease activity indices for rheumatoid arthritis: validation of a clinical activity score. Arthritis research \& therapy 7(4):R796-R806

6. Inoue E, Yamanaka H, Hara M, Tomatsu T, Kamatani N (2007) Comparison of Disease Activity Score (DAS)28-erythrocyte sedimentation rate and DAS28-C-reactive protein threshold values. Ann Rheum Dis 66(3):407-409

7. Smolen JS, Breedveld FC, Schiff MH, Kalden JR, Emery P, Eberl $G$ et al (2003) A simplified disease activity index for rheumatoid arthritis for use in clinical practice. Rheumatology (Oxford, England) 42(2):244-257

8. Pincus T, Bergman MJ, Yazici Y (2009) RAPID3-an index of physical function, pain, and global status as "vital signs" to improve care for people with chronic rheumatic diseases. Bulletin of the NYU hospital for joint diseases 67(2):211-225

9. Centola M, Cavet G, Shen Y, Ramanujan S, Knowlton N, Swan KA, Turner M, Sutton C, Smith DR, Haney DJ, Chernoff D, Hesterberg LK, Carulli JP, Taylor PC, Shadick NA, Weinblatt ME, Curtis JR (2013) Development of a multi-biomarker disease activity test for rheumatoid arthritis. PLoS One 8(4):e60635

10. Curtis JR, van der Helm-van Mil AH, Knevel R, Huizinga TW, Haney DJ, Shen Y, Ramanujan S, Cavet G, Centola M, Hesterberg LK, Chernoff D, Ford K, Shadick NA, Hamburger M, Fleischmann R, Keystone E, Weinblatt ME (2012) Validation of a novel multibiomarker test to assess rheumatoid arthritis disease activity. Arthritis Care Res 64(12):1794-1803

11. Heegaard C, Dreyer L, Egsmose C, Madsen OR (2013) Test-retest reliability of the disease activity score $28 \mathrm{CRP}$ (DAS28-CRP), the simplified disease activity index (SDAI) and the clinical disease activity index (CDAI) in rheumatoid arthritis when based on patient self-assessment of tender and swollen joints. Clin Rheumatol 32(10): 1493-1500

12. Uhlig T, Kvien TK, Pincus T (2009) Test-retest reliability of disease activity core set measures and indices in rheumatoid arthritis. Ann Rheum Dis 68(6):972-975

13. van Gestel AM, Prevoo ML, Van 't Hof MA, van Rijswijk MH, van de Putte LB, van riel PL (1996) Development and validation of the European League Against Rheumatism response criteria for rheumatoid arthritis. Comparison with the preliminary American College of Rheumatology and the World Health Organization/ International League Against Rheumatism Criteria. Arthritis Rheum 39(1):34-40

14. Zhao X, Qureshi F, Eastman PS, Manning WC, Alexander C, Robinson WH, Hesterberg LK (2012) Pre-analytical effects of blood sampling and handling in quantitative immunoassays for rheumatoid arthritis. J Immunol Methods 378(1-2):72-80
15. Eastman PS, Manning WC, Qureshi F, Haney D, Cavet G, Alexander C, Hesterberg LK (2012) Characterization of a multiplex, 12-biomarker test for rheumatoid arthritis. J Pharm Biomed Anal 70:415-424

16. Arvidson NG, Gudbjornsson B, Elfman L, Ryden AC, Totterman TH, Hallgren R (1994) Circadian rhythm of serum interleukin-6 in rheumatoid arthritis. Ann Rheum Dis 53(8):521-524

17. Straub RH, Cutolo M (2007) Circadian rhythms in rheumatoid arthritis: implications for pathophysiology and therapeutic management. Arthritis Rheum 56(2):399-408

18. Aletaha D, Neogi T, Silman AJ, Funovits J, Felson DT, Bingham CO 3rd et al (2010) 2010 Rheumatoid arthritis classification criteria: an American College of Rheumatology/European League Against Rheumatism collaborative initiative. Arthritis Rheum 62(9):2569-2581

19. Prevoo ML, Van 't Hof MA, Kuper HH, van Leeuwen MA, van de Putte LB, van riel PL (1995) Modified disease activity scores that include twenty-eight-joint counts. Development and validation in a prospective longitudinal study of patients with rheumatoid arthritis. Arthritis Rheum 38(1):44-48

20. Burmester GR, Weinblatt ME, McInnes IB, Porter D, Barbarash O, Vatutin M et al (2013) Efficacy and safety of mavrilimumab in subjects with rheumatoid arthritis. Ann Rheum Dis 72(9): $1445-1452$

21. Fraser Callum G (2012) Reference change values. Clin Chem Lab Med. p. 807

22. Curtis JR, Flake D, Weinblatt M, Shadick N, Ostergaard M, Hetland M, et al. (2018) Development of an adjusted multibiomarker disease activity score for rheumatoid arthritis that accounts for age, gender and adiposity with subsequent evaluation of ability to predict risk for radiographic damage. Rheumatology. submitted

23. Curtis JR, Chen L, Danila MI, Saag KG, Parham KL, Cush JJ (2017) Routine use of quantitative disease activity measurements among US rheumatologists: implications for treat-to-target management strategies in rheumatoid arthritis. J Rheumatol

24. Pincus T, Furer V, Keystone E, Yazici Y, Bergman MJ, Luijtens K (2008) Limitations of a quantitative swollen and tender joint count to assess and monitor patients with rheumatoid arthritis. Bulletin of the NYU hospital for joint diseases 66(3):216-223

25. Pincus T, Segurado OG (2006) Most visits of most patients with rheumatoid arthritis to most rheumatologists do not include a formal quantitative joint count. Ann Rheum Dis 65(6):820-822

26. Cheung PP, Gossec L, Mak A, March L (2014) Reliability of joint count assessment in rheumatoid arthritis: a systematic literature review. Semin Arthritis Rheum 43(6):721-729

27. Sokka T, Pincus T (2009) Joint counts to assess rheumatoid arthritis for clinical research and usual clinical care: advantages and limitations. Rheum Dis Clin N Am 35(4):713-722 v-vi

28. Kay J, Morgacheva O, Messing SP, Kremer JM, Greenberg JD, Reed GW, Gravallese EM, Furst DE (2014) Clinical disease activity and acute phase reactant levels are discordant among patients with active rheumatoid arthritis: acute phase reactant levels contribute separately to predicting outcome at one year. Arthritis research \& therapy 16(1):R40

29. Brown AK, Conaghan PG, Karim Z, Quinn MA, Ikeda K, Peterfy CG, Hensor E, Wakefield RJ, O'Connor PJ, Emery P (2008) An explanation for the apparent dissociation between clinical remission and continued structural deterioration in rheumatoid arthritis. Arthritis Rheum 58(10):2958-2967

30. Hambardzumyan K, Bolce R, Saevarsdottir S, Cruickshank SE, Sasso EH, Chernoff D, Forslind K, Petersson IF, Geborek P, van Vollenhoven RF (2015) Pretreatment multi-biomarker disease activity score and radiographic progression in early RA: results from the SWEFOT trial. Ann Rheum Dis 74(6):1102-1109 
31. Li W, Sasso EH, van der Helm-van Mil AH, Huizinga TW (2016) Relationship of multi-biomarker disease activity score and other risk factors with radiographic progression in an observational study of patients with rheumatoid arthritis. Rheumatology 55(2):357-366

32. Markusse IM, Dirven L, van den Broek M, Bijkerk C, Han KH, Ronday HK, Bolce R, Sasso EH, Kerstens PJSM, Lems WF, Huizinga TWJ, Allaart CF (2014) A multibiomarker disease activity score for rheumatoid arthritis predicts radiographic joint damage in the BeSt study. J Rheumatol 41(11):2114-2119

33. van der Helm-van Mil AH, Knevel R, Cavet G, Huizinga TW, Haney DJ (2013) An evaluation of molecular and clinical remission in rheumatoid arthritis by assessing radiographic progression. Rheumatology 52(5):839-846

34. Ino M, Ushiro K, Ino C, Yamashita T, Kumazawa T (1993) Kinetics of epidermal growth factor in saliva. Acta Otolaryngol Suppl 500:126-130
35. Langendonk JG, Pijl H, Toornvliet AC, Burggraaf J, Frolich M, Schoemaker RC et al (1998) Circadian rhythm of plasma leptin levels in upper and lower body obese women: influence of body fat distribution and weight loss. J Clin Endocrinol Metab 83(5):1706-1712

36. Perry MG, Kirwan JR, Jessop DS, Hunt LP (2009) Overnight variations in cortisol, interleukin 6, tumour necrosis factor alpha and other cytokines in people with rheumatoid arthritis. Ann Rheum Dis 68(1):63-68

37. Ward MM, Guthrie LC, Alba MI (2015) Clinically important changes in individual and composite measures of rheumatoid arthritis activity: thresholds applicable in clinical trials. Ann Rheum Dis 74(9): 1691-1696

38. Curtis JR, Yang S, Chen L, Pope JE, Keystone EC, Haraoui B, Boire G, Thorne JC, Tin D, Hitchon CA, Bingham CO III, Bykerk VP (2015) Determining the minimally important difference in the clinical disease activity index for improvement and worsening in early rheumatoid arthritis patients. Arthritis Care Res 67(10): $1345-1353$ 